\title{
Intrapericardial Extralobar Pulmonary Sequestration Presenting as a Prenatal Intrathoracic Mass
}

\author{
I. de Vreede $\cdot$ C. M. Bilardo $\cdot$ R. R. van Rijn • \\ S. A. B. Clur $\cdot$ H. A. Heij
}

Received: 20 February 2007 / Accepted: 25 October 2007/Published online: 6 March 2008

(C) The Author(s) 2008

\begin{abstract}
An intrathoracic mass, which persisted during the remaining pregnancy, was first seen during routine ultrasound examination performed at 20 weeks gestation. After birth, the child was asymptomatic. Echocardiography showed the mass to be located intrapericardially. The mass was electively resected via sternotomy 3 weeks after the birth. Microscopic examination showed normal lung tissue surrounded by pleura corresponding to the diagnosis of extralobar pulmonary sequestration. To the authors' knowledge, this is the earliest described detection of such a lesion. Furthermore, this article reports the unique finding of a feeding vessel from the right pulmonary artery.
\end{abstract}

I. de Vreede $(\square)$

Department of Paediatrics, Emma Children's Hospital,

Academic Medical Center, University of Amsterdam, 1105 AZ, Amsterdam, The Netherlands

e-mail: i.devreede@amc.uva.nl

C. M. Bilardo

Department of Obstetrics and Gynaecology, Academic Medical Center, University of Amsterdam, 1105 AZ, Amsterdam,

The Netherlands

R. R. van Rijn

Department of Paediatric Radiology, Emma Children's Hospital, Academic Medical Center, University of Amsterdam, 1105 AZ, Amsterdam, The Netherlands

\section{S. A. B. Clur}

Department of Paediatric Cardiology, Emma Children's

Hospital, Academic Medical Center, University of Amsterdam,

1105 AZ, Amsterdam, The Netherlands

\section{H. A. Heij}

Paediatric Surgical Center Amsterdam, Emma Children's

Hospital, Academic Medical Center, University of Amsterdam,

1105 AZ, Amsterdam, The Netherlands
Keywords Extrapulmonary · Intrapericardial · Prenatal $\cdot$ Sequestration

Fetal intrathoracic masses during antenatal ultrasound examinations are rare findings and may have similar ultrasonographic appearances, making it a challenge to determine the correct diagnosis before birth. We report a case of a neonate presenting with an intrathoracic mass at 20 weeks of gestation diagnosed as an intrapericardial extralobar pulmonary sequestration.

\section{Case Report}

A 39-week gestational age boy was born to a gravida 1, para 0 mother after spontaneous onset of labor. His birth weight was 2,770 g, and his Apgar score was 8 after 1 min and 8 after $5 \mathrm{~min}$. He had no respiratory problems.

The mother had been referred at 24 weeks of pregnancy to a tertiary center for prenatal diagnosis after a routine ultrasound examination at 20 weeks gestational age identified an intrathoracic mass measuring $2 \times 3 \times 2 \mathrm{~cm}$. The fetal echocardiogram performed at 24 weeks showed a structurally normal heart with the axis rotated to the left. The heart was surrounded by an echo-dense mass. Mild tricuspid regurgitation was present, and the vena cava superior was displaced laterally and posteriorly to the right.

Differential diagnosis included congenital cystic adenomatoid malformation (CCAM, small cyst type), congenital diaphragmatic hernia, and pulmonary sequestration. However, no feeding vessel was noted arising from the aorta. No other abnormalities were visualized, and the mass persisted throughout pregnancy. No signs of cardiac failure were noted. A retrospective review of the 
echocardiograms was able to identify a feeding vessel originating from the right pulmonary artery (Fig. 1).

An anteroposterior chest radiograph on the first day of life showed a widened mediastinum and an increased cardiothoracic ratio. The lungs were normally aerated, and 12 pairs of ribs were seen. The thoracic vertebrae were normally developed.

An ultrasound examination of the chest showed a thymus placed high within the thoracic cavity. Situated between the thymus and the heart, a mass of intermediate echogenicity was seen. One large feeding vessel, seeming to originating from the right pulmonary artery, was identified (Fig. 2).

An echocardiogram showed a structurally normal heart surrounded by an intrapericardial mass. Color Doppler imaging of the mass showed no flow except for the aforementioned vessel and its branches. Contrast-enhanced computed tomography (30 mAs, $120 \mathrm{Kv}, 2.5-\mathrm{mm}$ collimation) clearly showed a mass situated within the superior anterior and posterior mediastinum displacing the superior caval vein laterally (Fig. 3).

The large vessel arising from the right pulmonary artery was again identified. The mass showed heterogeneous enhancement. Within the mass, two small loculated areas of air were noted. A right heart catheterization confirmed the origin of the feeding artery from the right pulmonary artery (Fig. 4). The alpha-foeto-protein concentration was within the normal limits for the boy's age.

Three weeks after birth, the child underwent an elective median sternotomy and resection of the mass, which was draped around the heart and had its vascular supply from the right pulmonary artery. Systemic venous drainage of

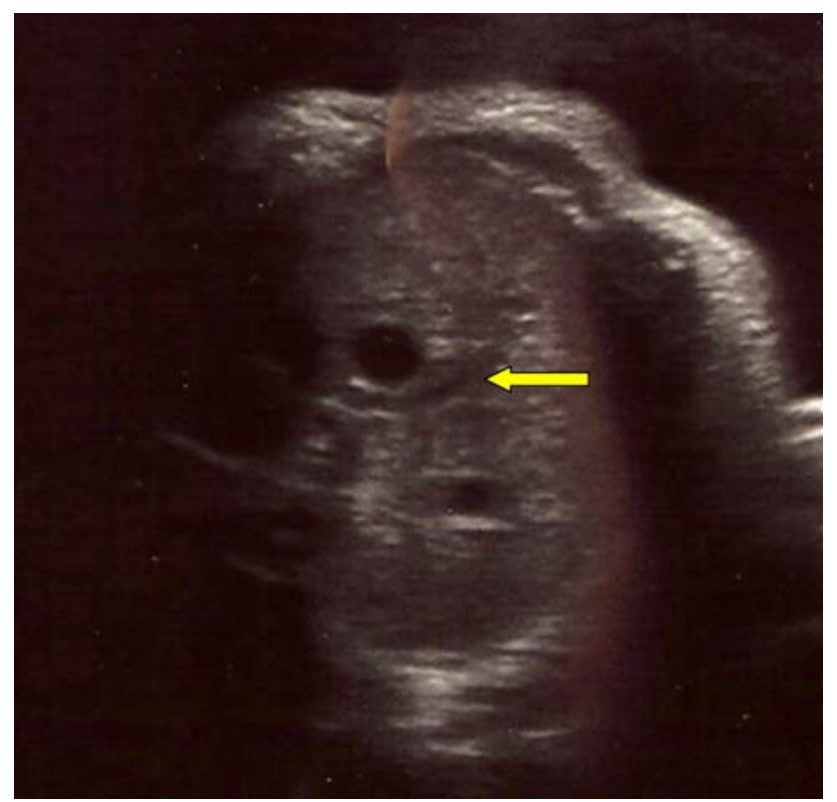

Fig. 1 Fetal echocardiogram showing the feeding vessel originating from the right pulmonary artery (arrow)

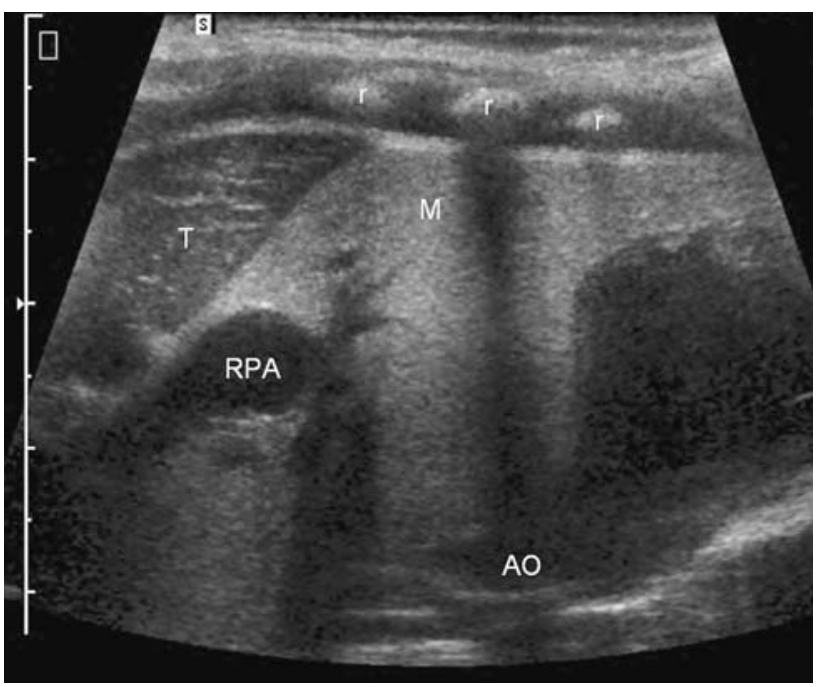

Fig. 2 Sagittal right parasternal ultrasound scan showing the thymus (T) and the mass $(\mathrm{M})$ in the superior mediastinum. The acoustic shadows are caused by rib cartilage ( $r$ ). The mass abuts the aorta (AO), and vessels arising from the right pulmonary artery (RPA) could be demonstrated

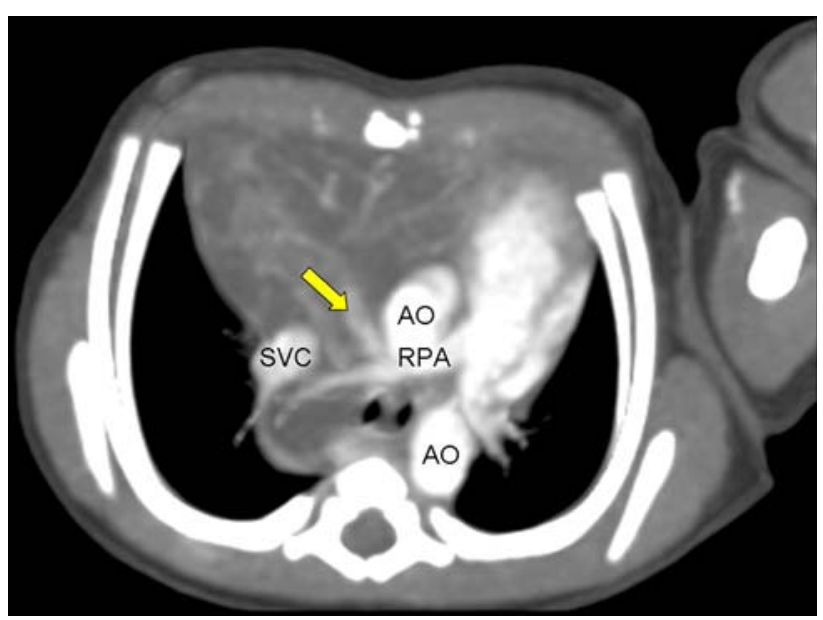

Fig. 3 A maximum intensity projection showing the mass displacing the superior caval vein (SCV) and abutting the aorta (AO). A vessel (arrow) arising from the right pulmonary artery (RPA) is noted

the sequester was found, which resulted in a right-to-right shunt. There was a connection with the trachea through an atretic side branch of the trachea. In the proximity of the trachea, a cystic lesion $0.5 \mathrm{~cm}$ diameter was found and removed.

After an uneventful recovery, the child was discharged home on postoperative day 5. Histologic examination showed a mass with a maximum diameter of $7 \mathrm{~cm}$ and a width of $2 \mathrm{~cm}$, macroscopically and microscopically identified as lung tissue. Therefore, the final diagnosis of an intrapericardial extralobar lung sequestration was made. After 2 years of follow-up evaluation, the child was in good health. 


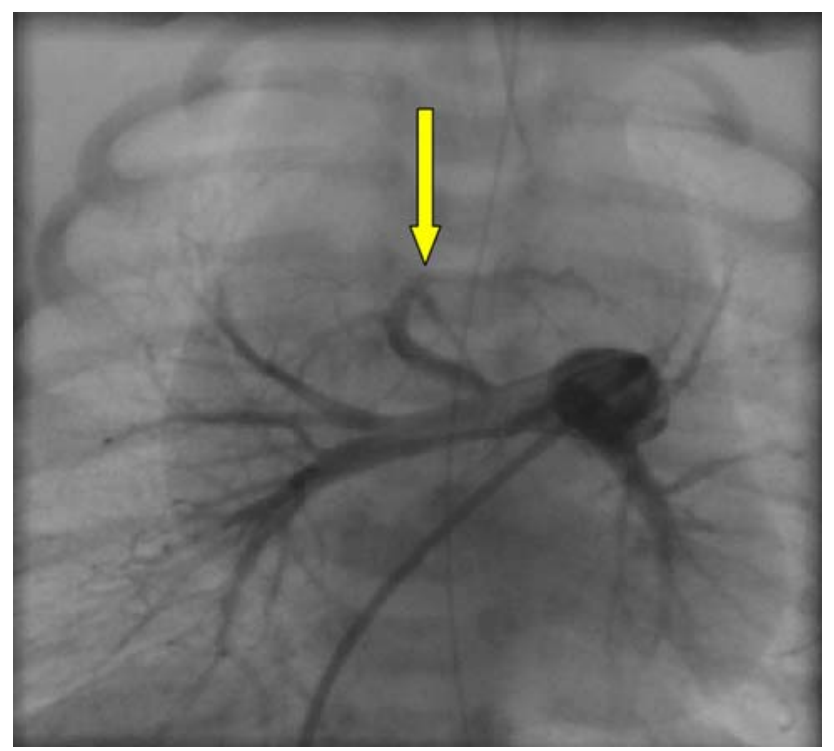

Fig. 4 Pulmonary angiogram showing the feeding artery originating from the right pulmonary artery in a frontal projection. Arrow indicates the feeding vessel

\section{Discussion}

Intrathoracic masses are uncommon congenital anomalies. However, because of the potentially disastrous consequences resulting from some of these intrathoracic masses, early diagnosis is of critical importance to allow sufficient time for the neonatologist and pediatric surgeon to prepare for the birth of a potentially very ill child. The differential diagnosis of an intrathoracic mass seen at this age includes teratoma, CCAM, intra- or extralobar pulmonary sequestration, hemangioma, and neuroblastoma.

In early pregnancy, the most likely diagnosis of a hyperechogenic intrathoracic mass is a CCAM. The main differentiation between a CCAM and an extrapulmonary sequestration is based on identification of a systemic artery arising from the aorta feeding the fetal lung mass. When the feeding vessel is not seen on color Doppler, an extrapulmonary sequestration can easily be misdiagnosed as a CCAM. As a unique finding in the reported case, the blood supply was from the right pulmonary artery.

Recent studies show that antenatally, both magnetic resonance imaging (MRI) and three-dimensional power Doppler ultrasound can be helpful in establishing the diagnosis of an extrapulmonary sequestration [5, 7]. However, differentiation from a teratoma still may not be possible until after resection [5, 7]. After birth, the diagnosis is made by ultrasound or contrast-enhanced computed tomography.

To our knowledge, only four cases of intrapericardial extralobar pulmonary sequestration diagnosed at neonatal age have been described [2, 3, 7, 8]. One case was suspected antenatally at a gestational age of 29 weeks [7].
Notably, another case involved the diagnosis of an intrapericardial sequestration in a 27-year-old adult [1].

Interestingly, in all the previously reported cases, the intrapericardial extralobar pulmonary sequestrations were surrounded by a pericardial effusion, in contrast to the case described in this report. Signs of congestive heart failure or cardiac tamponade were noted in none of the cases after birth. Microscopic examination can vary between completely normal lung tissue and degenerated lung tissue to a cystic accessory lobe. Intrapericardial extralobar pulmonary sequestration has been associated with bronchogenic cysts $[2,3,6]$. Theoretically, an intrapericardial extralobar pulmonary sequestration must develop between weeks 6 and 7 after conception. The embryology of these lesions has been described previously [4]. Postnatal treatment of an intrapericardial extralobar pulmonary sequestration consists of resection to rule out the possibility of a teratoma and to prevent possible congestive heart failure in future life.

In conclusion, our case report describes the earliest detection of an intrathoracic mass and highlights the difficulty of establishing the prenatal diagnosis of an intrapericardial pulmonary sequestration. The presence of a feeding vessel from the right pulmonary artery instead of a feeding vessel from the aorta makes this case unique. A prenatal diagnosis is of utmost importance to enable adequate reception and management of a potentially ill neonate after birth.

Open Access This article is distributed under the terms of the Creative Commons Attribution Noncommercial License which permits any noncommercial use, distribution, and reproduction in any medium, provided the original author(s) and source are credited.

\section{References}

1. Chul Min A, Hyung Jung K, Hyun Keun C, et al. (1991) A case of intrapericardal extralobar pulmonary sequestration. Korean $\mathrm{J}$ Internal Med 6:85-89

2. Hayashi AH, McLean DR, Peliowski A, et al. (1992) A rare intrapericardial mass in a neonate. J Pediatr Surg 10:1361-1363

3. Levi A, Findler M, Dolfin T, et al. (1990) Intrapericardial extralobar pulmonary sequestration in a neonate. Chest 98:1014

4. Moore KL (1982) The developing human. 3rd ed. Saunders, Philadelphia, PA pp 229-230

5. Ruano R, Benachi A, Aubry MC, et al. (2005) Prenatal diagnosis of pulmonary sequestration using three-dimensional power Doppler ultrasound. Ultrasound Obstet Gynaecol 25:128-133

6. Savic B, Birtel FJ, Thalen W, et al. (1979) Lung sequestration report of seven cases and review of 540 published cases. Thorax 34:96-101

7. Wax JR, Pinete MG, Landes A, et al. (2002) Intrapericardial extralobar pulmonary sequestration: Ultrasound and magnetic resonance prenatal diagnosis. Am J Obstet Gynaecol 187:17131714

8. Yildiz K, Ozcan N, Cebi M, et al. (2005) Intrapericardial extralobar pulmonary sequestration: Unusual cause of hydrops fetalis. J Ultrasound Med 24:391-393 